\title{
Ethical considerations of COVID-19-related adjustments to clinical research
}

\author{
Unexpected direct and indirect risks of participating in clinical trials have emerged during COVID-19 that \\ investigators and institutional review boards may not be sure how to investigate. How should existing guidance \\ and ethical frameworks for clinical trials be applied in a pandemic setting?
}

\author{
Nina S. Hsu, Saskia Hendriks, Khara M. Ramos and Christine Grady
}

T he COVID-19 pandemic continues to affect clinical research considerably, forcing policymakers and institutions to make difficult decisions about delaying, continuing and starting research while protecting public health. Delaying research can affect its social value and possible benefits for participants. As clinical studies re-start - and for those that never halted - most investigators have to adjust their procedures and protocols to protect participants, staff and public health and to adhere to institutional COVID-19 policies. Both the US National Institutes of Health (NIH) and the US Food and Drug Administration (FDA) have endorsed the need for such adjustments ${ }^{1,2}$, which may include tele-visits instead of in-person visits, reducing visit frequency, and restricting how many staff members interact with participants. Some of these changes may affect ethically meaningful trial elements, such as risks and benefits for participants, or the trial's social value ${ }^{2,3}$.

We learned from investigators and bioethicists during two meetings organized by the NIH BRAIN (Brain Research through Advancing Innovative Neurotechnologies) Initiative $e^{4,5}$ that these procedural or protocol changes raise some key ethical challenges investigators face due to COVID-19.

First, the pandemic introduces a new risk: possible contraction of COVID-19 during participation in a study. The pandemic also affects certain benefits and risks that are not always considered, including the value of socialization with research staff, for socially isolated participants. How should investigators navigate these altered benefits and risks?

Second, what should investigators do if they anticipate that COVID-19-related changes may substantially impact their study's ethically salient elements, such as the risk-benefit profile? Although the experiences of neurotechnology researchers provided the basis for this Comment, we anticipate, on the basis of reports from other fields $s^{3,6}$, that these challenges are more broadly relevant in clinical research.

In this Comment, we consider these ethical questions in the context of existing ethical frameworks and provide points for investigators and institutional review boards (IRBs) to consider in navigating these challenges. Finally, we reflect on how these considerations may facilitate clinical research preparedness for future pandemics.

\section{Changes that affect indirect risks and benefits}

Some investigators reported that healthy volunteers are increasingly interested in paid study enrollment, potentially due to pandemic-induced financial hardships. Other investigators reported concerns about their socially isolated participants' mental health, as home visits by the study team were curbed to reduce the risk of SARS-CoV-2 transmission. Some investigators described the challenges of remote care for participants who lack access to certain technologies or are uncomfortable with using them ${ }^{7}$. Finally, some investigators recounted that participants requested early release from extended hospital admissions because of COVID-19 restrictions on visitors. These benefits (payment and social contact) or risks (isolation and infection risk) may matter to participants, but how should investigators navigate them?

Bioethicists describe these types of research benefits and risks as 'indirect' or 'collateral': those that arise from participation in a study but are not linked to the study intervention or procedures ${ }^{8-10}$. Other examples of collateral risks and benefits include payment for parking, complementary medical care, or the gratification of contributing to science.

Although this recommendation is sometimes challenged ${ }^{11,12}$, bioethics guidance suggests that indirect benefits not be included in an IRB's risk-benefit evaluation, because indirect benefits might distort the risk-benefit profile, which would allow the conduction of trials with highly unfavorable clinical risk-benefit profiles for participants $^{8,13}$. Similarly, IRBs scrutinize how indirect benefits are described in informed-consent documents, to prevent undue influence on patients to enroll against their best interests ${ }^{11}$.

While limited bioethics guidance addresses indirect risks, including indirect risks but not indirect benefits in the riskbenefit analysis may skew this evaluation. In our experience, indirect risks (like indirect benefits) are therefore generally not included in an IRB's risk-benefit evaluation. In these COVID-19 cases, adhering to these general guidance and practices seems reasonable, such that neither IRBs nor investigators need to include indirect benefits or risks in evaluating study acceptability or reflect them in the research study's informed-consent process.

Nonetheless, indirect risks and benefits can influence participants' decision-making about study participation ${ }^{11}$. Investigators may consider minor adaptations to study procedures to optimize indirect benefits or mitigate risks that may be meaningful for participants (e.g., offering resources for psychosocial support) ${ }^{6}$. Even if it is not part of the informed-consent process, investigators might discuss new or changing indirect risks and benefits with their participants.

Most trials at present will entail a new risk for participants: exposure to COVID19 during engagement in research-related activities (e.g., clinic visits). This risk is 'new' because COVID-19 is new, but is not unique because exposure to infectious diseases (e.g., influenza) has always been a risk of visiting hospitals and public spaces.

We suggest that in general, exposure to COVID-19 is an indirect research risk, as it is not specifically linked to the intervention under study. Therefore, consistent with the consideration of other indirect risks and benefits, IRBs do not need to incorporate this risk in assessing whether a study's 
risk-benefit profile is acceptable. However, compared with other infectious diseases to which people could be accidentally exposed as part of trial participation, COVID-19 can be relatively easily transmitted, can be more severe, and/or has few effective treatments. The risk of acquiring COVID-19 while engaging in research is therefore certainly relevant and potentially meaningful for participants. We recommend that investigators and institutions communicate to participants about risk-mitigation measures, which will allow participants to assess their risk of COVID-19 and make their own choices about (continued) research participation.

\section{Changes that affect direct risks and benefits, social value or scientific integrity}

Some changes in study procedures or protocols that investigators described have potentially more meaningful effects on the ethics of their trial. For example, if more participants request an early discharge from the hospital after surgery because of COVID-19 risks and no-visitors policies, this could limit safety monitoring, which might potentially affect the procedure's risks and the study's scientific integrity. Similarly, missed time points for data collection because of COVID-19 restrictions can affect a study's scientific integrity and limit the value of the knowledge collected (i.e., social value). Sometimes the risk of COVID-19 is directly related to the study intervention (e.g., trials studying immunomodulatory drugs, which may now involve greater direct risks) ${ }^{3}$.

Many such changes are beyond the investigators' control and might affect the study's overall evaluation only rarely. However, considering whether the altered trial's risks are still justified by its benefits (for participants and/or society) is important. Disproportional risks could justify pausing a trial until the pandemic has receded $^{3}$. As always, when a study changes in potentially meaningful ways, IRBs are tasked with overseeing whether the study still complies with federal research regulations (such as the US Code of Federal Regulations rules 45 CFR 46 and 21 CFR 56). IRBs can also help identify when modifications to the informed-consent process are warranted.

Clearly, not all possible COVID19-related changes are within an IRB's purview, so investigators are crucial in identifying any changes that are potentially ethically consequential. We recommend that investigators carefully consider whether what IRBs assess may have been altered, including the risk-benefit profile for participants, the study's scientific and social value, and what is disclosed to participants during the informed-consent process. When in doubt, investigators should check with their IRB. In some cases, investigators may need to inform the FDA of protocol changes $^{2}$.

We agree with the FDA guidance that pandemic-induced delays in research will usually have limited effects on the overall evaluation of a trial ${ }^{2}$. While temporarily halting a trial may delay its social value, it is unlikely to alter the study's overall assessments unless new developments challenge the rationale for conducting the study. A trial's risks and benefits for individual participants may change because the participants had to wait for a procedure, but this generally will not modify the trial's overall risk-benefit profile ${ }^{2}$.

\section{Preparing for future pandemics}

Unfortunately, the world is likely to be faced with other pandemics in the future ${ }^{14}$. The experience with COVID-19 should be leveraged to improve preparedness for future pandemics. In the context of clinical research that is not directly related to infectious-disease outbreaks, a key component of pandemic preparedness may involve appreciation of the effects that pandemics may have on research, and best practices for how to deal with those changes. We anticipate that many, if not all, of the COVID-19-related adjustments to clinical research discussed in this Comment would be relevant for other pandemics involving an infectious disease with easy human-to-human transmission (e.g., airborne transmission of an influenza virus) that warrants social distancing to limit the spread of the disease. Other types of pandemics, such as those that involve a different mortality rate or mode of transmission, may not affect clinical research in the same way. In these types of cases, non-pandemic-related clinical research may also need to be delayed to free up resources and personnel for healthcare systems. However, for ongoing studies, there may be less reason to adjust research protocols or procedures to protect participants, staff and the public from transmission, or for the ethically salient study features to change.

There are limitations to the ability to prepare for future pandemics, given the uncertainty about the infectious agents involved $^{14}$. It is also necessary to consider how to invest limited resources in pandemic preparation without unduly burdening research in non-pandemic times with planning for scenarios that can be dealt with 'just in time' or may never happen. Nonetheless, there are some preparedness measures that clinical researchers and research institutions can endeavor toward before the next pandemic.

First, as noted above, preserving institutional awareness about the issues faced during this pandemic would allow investigators to incorporate lessons learned from this one into future decisions. Second, the current pandemic has increased the use of telehealth, including telehealth for research purposes, and experts anticipate a sustained higher level of telehealth after the pandemic ends ${ }^{15}$. A full assessment of the strengths and weaknesses of telehealth and in-person visits, including clinical and research outcomes ${ }^{15}$, is important to ensure that using telehealth - whether during a new pandemic or during non-pandemic times - will not compromise the scientific integrity or risk-benefit profile of studies. Finally, training and educational materials that assist investigators in their ability to assess whether protocol and/or procedural modifications are sufficiently ethically consequential to warrant IRB involvement can help with preparedness for research disruptions, whether in a pandemic or otherwise.

COVID-19 raises more ethical challenges in research than those addressed here ${ }^{16}$; researchers should continue to identify and address these ethical challenges while taking stock of best practices to prepare for possible future pandemics.

\section{Nina S. Hsu ${ }^{1,3 凶}$, Saskia Hendriks ${ }^{1,2,3}$,} Khara M. Ramos ${ }^{1}$ and Christine Grády ${ }^{2}$ ${ }^{1}$ National Institute of Neurological Disorders and Stroke, National Institutes of Health, Bethesda, MD, USA. ${ }^{2}$ Department of Bioethics, NIH Clinical Center, Bethesda, MD, USA. ${ }^{3}$ These authors contributed equally: Nina S. Hsu, Saskia Hendriks.

$\bigotimes_{e-m a i l: n i n a . h s u @ n i h . g o v}$

Published online: 25 January 2021 https://doi.org/10.1038/s41591-020-01216-2

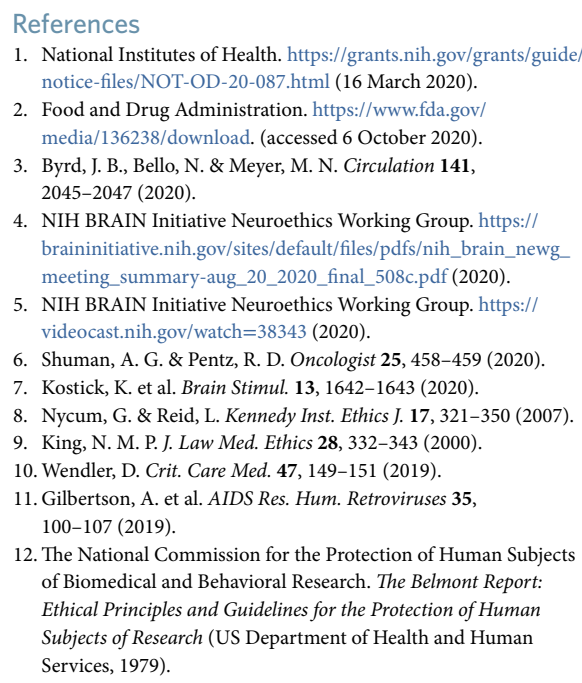


13. Emanuel, E. J., Wendler, D. \& Grady, C. J. Am. Med. Assoc. 283, 2701-2711 (2000)

14. Taubenberger, J. K., Morens, D. M. \& Fauci, A. S. J. Am. Med. Assoc. 297, 2025-2027 (2007)

15. Wosik, J. et al. J. Am. Med. Inform. Assoc. 27, 957-962 (2020)

16. Kim, S. Y. H. \& Grady, C. Neurology $94,1007-1008$ (2020).
Acknowledgements

The authors thank the members of the NIH BRAIN Initiative Neuroethics Working Group, meeting presenters (M. Franceschini, L. Hochberg and S. Sheth) and the participants and organizers of the neuroethics-focused session at the BRAIN Initiative Investigators Meeting. This research was supported in part by the Intramural Research
Program of the NIH. The views expressed are the authors' own and do not necessarily reflect those of the National Institutes of Health, the Department of Health and Human Services, or the United States government.

Competing interests

The authors declare no competing interests.

\title{
To tackle diabetes, science and health systems must take into account social context
}

\author{
An increasing amount of publications are recognizing that a person's risk of diabetes and diabetes outcomes are \\ influenced largely by social determinants of health. This renewed understanding of disease should influence health \\ provision and diabetes research, but will it?
}

\section{Jacqueline A. Seiglie, Devaki Nambiar, David Beran and J. Jaime Miranda}

\begin{abstract}
$\mathrm{n}$ a year marked by the centenary of the discovery of insulin ${ }^{1}$ and outstanding scientific progress in the therapeutic management of diabetes mellitus ${ }^{2,3}$, these advances have been contrasted by the sobering disparities that, although present before the COVID-19 pandemic, have been unmasked by this crisis for people living with diabetes. While diabetes is now well recognized as an important biological risk factor for poor COVID-19 outcomes, the disproportionate impact of this pandemic on socially vulnerable people with diabetes has laid bare the profound importance of the social determinants of health in the tackling of health inequalities ${ }^{4,5}$. These disparities are particularly alarming when one considers that among the nearly half-billion people with diabetes globally, three out of every four people with diabetes live in low- and middle-income countries (LMICs) ${ }^{6}$. The renewed attention on the social determinants of health in relation to diabetes in the wake of the COVID-19 pandemic has revealed the sheer complexity of tackling health inequalities and the inadequacy of existing metrics, frameworks and approaches ${ }^{7}$. The question is: amid the astounding scientific progress accomplished in the management of diabetes, what does a social-determinants framework add to the field, particularly in LMICs?

It has long been recognized that health is strongly influenced by social determinantsthe conditions in which people are born, grow, live, work, and age, including the health system ${ }^{8}$. In fact, the concept of the social determinants of health was woven
\end{abstract} into the very foundations of modern public health, which at the turn of the 19th century inspired pioneers such as Rudolf Virchow to posit that social medicine was inextricably intertwined with the politics of social justice $^{9,10}$. Further evidence on the strong linkage between the social determinants and their impact on disease emerged throughout the 20th century ${ }^{11}$ and, in 2008, culminated in the report of the World Health Organization's Commission on the Social Determinants of Health ${ }^{8}$, which called for a global movement to recognize their importance in the tackling of health inequalities, with the goal of closing the health gap within a generation. Yet more than a decade since that call for action was put forth, the COVID-19 crisis has abruptly highlighted the lack of progress achieved in tackling inequalities, making visible the linkages and the gross weaknesses of living conditions, health and well-being, and the impact of this on multiple facets of long-term chronic conditions ${ }^{12,13}$.

In their timely article 'Social determinants of health and diabetes: a scientific review, published in Diabetes $\mathrm{Care}^{4}$, the authors provide an overview of key definitions and social-determinant frameworks of diabetes and outline detailed recommendations for their implementation into community sectors, diabetes research and clinical practice in the USA. The review builds on pragmatic scientific and consensus statements published before the COVID-19 pandemic ${ }^{14,15}$ and categorizes social determinants of diabetes into the following five domains: socioeconomic status; neighborhood and physical environment; food environment; healthcare; and social context ${ }^{4}$. Each of these domains encompass aspects of life circumstances that influence how a person's risk of diabetes and diabetes outcomes are shaped-a phenomenon that occurs largely outside of the health system $^{16}$. While there is robust evidence to support this framework, much of this evidence has been generated in high-income contexts. Less is known about the social determinants of diabetes in LMICs, which are highly heterogeneous and may be subject to additional societal pressures, such as socio-political and protracted conflicts ${ }^{17}$, environmental pollutants ${ }^{18}$, and corporate and commercial power ${ }^{19,20}$, as well as constrained health systems with limited capacity to manage chronic conditions that rely on continuity of care ${ }^{21}$.

Whereas much of the scientific progress in diabetes has gravitated toward the domain of pharmacological interventions, diabetes 'exists' within a much broader context, with factors that influence its development throughout the life course. For instance, an adverse intrauterine and postnatal environment may influence the development of insulin resistance and the onset of metabolic disease later in life $^{22}$. When these early life disadvantages are coupled with social and commercial determinants that catalyze the development of overweight and obesity ${ }^{19}$, the cumulative burden of these life-course circumstances, which disproportionately affect vulnerable segments of the population, can be difficult to overcome even within equitable health systems. Notably, solutions for mitigating the risk of diabetes may very well lie outside the sphere of health systems, as 